\title{
Current Research Progress on Long Noncoding RNAs Associated with Hepatocellular Carcinoma
}

\author{
Haihong Shi $\mathbb{D},{ }^{1}$ Yuxin $\mathrm{Xu}^{2}{ }^{2}$ Xin $\mathrm{Yi}^{1},{ }^{1}$ Dandan Fang, ${ }^{3}$ and Xia Hou $\mathbb{D}^{1,4}$ \\ ${ }^{1}$ Department of Biochemistry and Molecular Biology, Jiamusi University School of Basic Medicine, Jiamusi, \\ Heilongjiang 154007, China \\ ${ }^{2}$ Jiamusi Maternal and Child Health Hospital, Jiamusi, Heilongjiang 154001, China \\ ${ }^{3}$ Hospital of Traditional Chinese Medicine of Qiqihar, Qiqihar, Heilongjiang 161000, China \\ ${ }^{4}$ Department of Physiology, Wayne State University School of Medicine, Detroit, MI 48201, USA \\ Correspondence should be addressed to Xia Hou; hou_xia@hotmail.com
}

Received 6 December 2018; Revised 20 February 2019; Accepted 10 March 2019; Published 24 June 2019

Guest Editor: Zhigang Ren

Copyright (C) 2019 Haihong Shi et al. This is an open access article distributed under the Creative Commons Attribution License, which permits unrestricted use, distribution, and reproduction in any medium, provided the original work is properly cited.

Hepatocellular carcinoma (HCC) is the second leading cause of mortality among cancers. It has been found that long noncoding RNAs (lncRNAs) are involved in many human cancers, including liver cancer. It has been identified that carcinogenic and tumor-suppressing lncRNAs are associated with complex processes in liver cancer. These lncRNAs may participate in a variety of pathological and biological activities, such as cell proliferation, apoptosis, invasion, and metastasis. Here, we review the regulation and function of lncRNA in liver cancer and evaluate the potential of lncRNA as a new goal for liver cancer.

\section{Introduction}

Hepatocellular carcinoma (HCC), the most common form of liver cancer, is involved in $90 \%$ of primary liver cancers. In the last few decades, liver cancer has become the most important commonly diagnosed tumor type worldwide. It is also considered to be the most lethal cancer, related to approximately $34 \%$ of all malignancies $[1,2]$. HCC is a highly invasive and fatal type of tumor that is often involved in relapse and metastasis, and the prognosis is poor. The incidence of liver cancer is related to a variety of risk factors, such as hepatitis B virus (HBV) and hepatitis $\mathrm{C}$ virus (HCV) infections, alcoholic cirrhosis, smoking, and aflatoxin B1 intake [3]. However, the molecular mechanism of the occurrence and development of liver cancer is complicated, which is related to different processes, e.g., cell cycle dysregulation, apoptosis, tumor cell invasion, and metastasis [4]. Accumulating proofs show that long noncoding RNA (lncRNA) expression is altered in liver cancer and involved in tumorigenesis [5].

In recent decades, most researches concerning the relationship between tumorigenesis and human genes have focused on structural genes and their related regulatory sequences. However, some studies show that noncoding sequences of human genes play crucial roles in tumorigenesis. The human genome contains approximately 3 billion base pairs, of which less than $2 \%$ encode proteins, whereas the remaining $~ 98 \%$ of the genome consists of non-proteincoding sequences. RNAs that cannot be translated into proteins are called noncoding RNAs (ncRNAs), which include lncRNA, siRNA, miRNA, and other types [6]. LncRNA consists of more than 200 nucleotides without protein-coding potential but with gene regulatory functions. LncRNAs are classified based on the related locations of their protein-encoding genes in the genome, including (1) sense, (2) antisense, (3) bidirectional, (4) intronic, and (5) intergenic (Figure 1). This positional relationship is helpful in predicting the lncRNA function. LncRNA is involved in the proliferation, migration, invasion, apoptosis, angiogenesis, and drug resistance of tumor cells, although it was previously considered as "transcriptional noise" [7-9]. LncRNA is also related to the regulation of biological functions and gene expression under physiological and pathological conditions [10]. However, only a few functional 


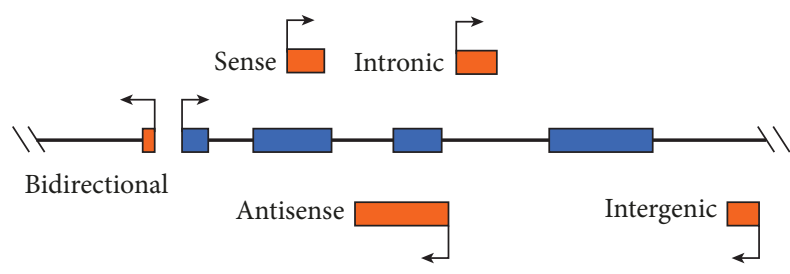

FIgURE 1: Based on the location of the lncRNA on the genome, it can be divided into five types: (1) sense, (2) antisense, (3) bidirectional, (4) intronic, and (5) intergenic. The coding RNA and noncoding RNA exons are shown in blue and red, respectively.

IncRNAs have been well characterized to date; several mechanistic topics of IncRNA function have been reviewed elsewhere $[11,12]$. In this review, a summary of the four well-known molecular functions of lncRNAs is shown (Figure 2): (1) signal function-lncRNA has the function of stimulating the combination of transcription factors, suggesting that it may act as a signal molecule regulating the expression of genes; (2) decoy function-transcription of a class of lncRNAs can then bind to and titrate protein or RNA targets without performing any other functions. (these lncRNAs may negatively regulate the expression of their targets by acting as molecular bait); (3) manipulation function-lncRNAs can recruit the chromatin-modifying enzyme to regulate or control the positioning of these enzymes close to or away from the target gene; and (4) scaffolding function-lncRNAs can aggregate with many proteins to form a nuclear protein complex, which is involved in the modification of histones. LncRNAs have been proven to play an essential biological role in carcinogenesis by regulating gene expression, such as carcinogenic and antitumor functions. LncRNAs can be used as a criterion for the diagnosis and prognosis of hepatocellular carcinoma [13-16].

In this review, we mainly discuss HCC-associated lncRNAs. Here, we summarized the differences in the expression of IncRNAs in HCC; then, we reviewed the participation of lncRNAs in HCC cell proliferation, apoptosis, and migration. Finally, we discussed the prospect of lncRNAs as potential biomarkers and therapeutic targets for HCC.

\section{Molecular Mechanisms of LncRNAs in HCC}

LncRNA is involved in the development of various molecular mechanisms of hepatocellular carcinoma: epigenetic regulation, the regulation of DNA damage and cell cycle progression, microRNA regulation, signal transduction pathways, and hormone-induced cancer [17]. In liver cancer, lncRNAs are used as transcriptional regulatory molecules for oncogenes and tumor suppressor genes [17]. For instance, IncRNA HOTAIR overexpression is associated with the development of liver cancer. In contrast, the lncRNA TARID can prevent cancer formation by causing the demethylation of tumor suppressor gene promoters [18]. Epigenetic regulation refers to genetic phenotypes and genetic changes in gene expression, including DNA methylation, histone modification, and chromatin remodelling, and does not result in any changes in DNA sequencing. Studies show that a crucial role in liver cancer development is that lncRNAs are accomplished by epigenetic regulation. LncRNAs can regulate gene expression through epigenetic regulation, transcriptional and posttranscriptional regulation, etc. LncRNAs participate in various biological processes in liver cancer cells, such as proliferation, differentiation, and apoptosis [19]. Besides, lncRNAs are involved in the regulation of a variety of epigenetic complexes, thus resulting in the activation and inactivation of genes. For example, lncRNA TCF7 is highly expressed in HCC cells and plays an important role in the maintenance of the self-renewal ability of the liver cancer stem cell [20]. The TCF7 gene is activated by recruiting SWI/SNF complexes to the gene and produces IncRNA TCF7, which can activate the Wnt signalling pathway and lead to the occurrence of hepatocarcinoma. The repair of DNA damage and the regulation of cell cycle checkpoints are important to maintain cell integrity. LncRNAs are also involved in DNA damage repair and in the regulation of physiological or pathological processes such as cell cycle, through which lncRNAs can regulate the occurrence and development of tumors [20]. The p53 gene, a tumor suppressor gene, also owns a robust ability to encode transcription factors in cells, which is at a low expression level under normal conditions. p53 can be activated by different signalling pathways under cellular stresses such as DNA damage, which results in cell cycle arrest, apoptosis or fading by enhancing the transcription of multiple downstream genes, maintaining cell genome integrity, and clearing damaged cells [21, 22]. As an example, lncRNA-p21 recruits ribonucleoprotein hnRNP-k to promote P21 transcription, a key molecule regulating the p53 signalling pathway. Downregulation of lincRNA-p21 causes losing control of G1/S checkpoints and leads to enhanced cell proliferation [23]. Furthermore, the mechanism of lncRNAs regulating microRNAs is that microRNAs (miRNAs) are related to the development of many diseases, including liver cancer [24]. miRNAs can bind to lncRNA sponges to inhibit gene expression and protein synthesis [25]; therefore, they affect the function of the cell, e.g., IncRNA XIST promotes HCC proliferation and inhibits apoptosis by regulating miR-139-5p/PDK1/AKT axis [26]. Finally, lncRNAs take part in signalling pathways and hormonal regulation. Evidence shows that liver cancer development is associated with the abnormal activation of signalling pathways. The role of lncRNAs in these signalling pathways is an essential part of the mechanism of liver cancer. Therefore, future studies on lncRNAs are also expected to find candidate drugs for the treatment of liver cancer. Studies have confirmed the roles of transforming growth factor beta (TGF- $\beta$ ), the AKT signalling pathway, and the Wnt signalling pathway in tumor development [27-29]. For example, TGF- $\beta$ promotes liver cancer cell metastasis via lncRNA-ATB. Abnormal AKT signalling leads to increased expression of lncRNA PTTG3P and promotes proliferation and migration of hepatoma cells.

\section{LncRNAs in HCC}

3.1. Dysregulation of LncRNAs in HCC. LncRNAs play irreplaceable roles in the progression of HCC because lncRNAs are known to be involved in the regulation of tumor 


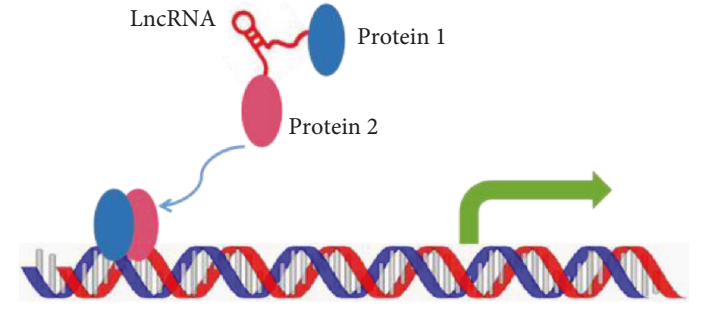

(a) Signal

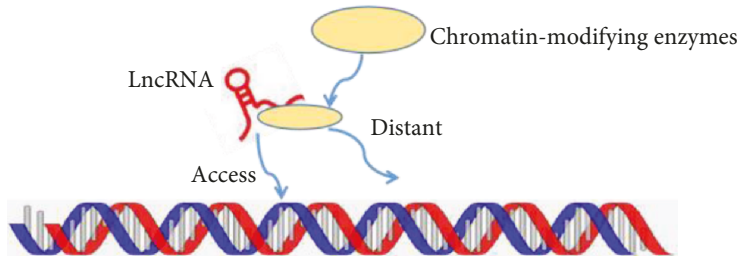

(c) Guide

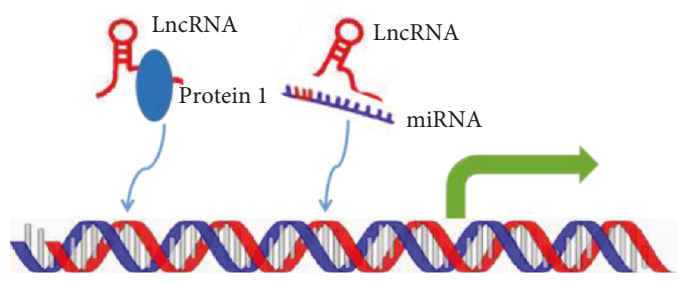

(b) Decoy

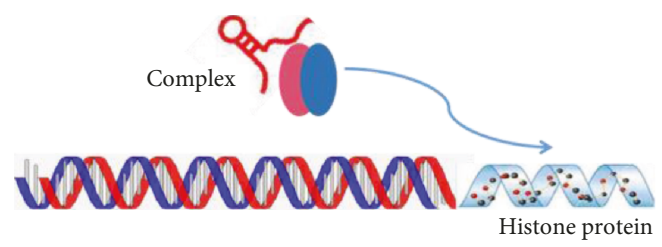

(d) Scafffolf

FIGURE 2: Four typical molecular functions of lncRNA: (a) LncRNAs can be used as molecular signalling mediators to regulate the expression of certain genes together with specific transcription factors or chromatin modifiers. (b) LncRNAs can bind to and titrate the expression of proteins or RNA, which indirectly play a variety of biological functions. (c) LncRNAs recruit chromatin-modifying enzymes that can enter or leave the target gene. (d) LncRNAs can pool multiple proteins to form ribonucleoprotein complexes and affect histone modifications.

TABLE 1: Dysregulated long noncoding RNAs (lncRNAs) associated with HCC.

\begin{tabular}{|c|c|c|c|c|}
\hline LncRNAs & Expression & Affected target genes and pathways & $\begin{array}{l}\text { Affected clinicopathological characteristics } \\
\text { of HCC }\end{array}$ & References \\
\hline HOTAIR & Upregulated & $\begin{array}{c}\text { HOXD/VEGF/MMP-9/PRC2/H3K27/rbm38/ } \\
\text { Bmi-1/P14/P16 }\end{array}$ & TNM stage, distant metastasis & {$[31-34]$} \\
\hline HULC & Upregulated & P18/PRKACB/CREB & TNM stage, intrahepatic metastases & {$[38,39,54]$} \\
\hline H19 & Upregulated & Cdc25A/E2F1/hnRNP U/PCAF/DMC/ZEB1/2 & & {$[51,55]$} \\
\hline URHC & Upregulated & ZAK & Tumor size, tumor number & [9] \\
\hline ROR & Upregulated & TGF- $\beta /$ PDK1/P53 & & {$[56]$} \\
\hline PVT1 & Upregulated & TGF- $\beta / \mathrm{NOP} 2$ & $\begin{array}{l}\text { AFP level, tumor size, tumor number, } \\
\text { tumor stage }\end{array}$ & {$[14,57]$} \\
\hline PTTG3P & Upregulated & PPTG1/AKT signalling & Tumor size, TNM stage & {$[43]$} \\
\hline XIST & Upregulated & miR-139-5p/PDK1/AKT signalling & Tumor size & {$[26]$} \\
\hline DBH-AS1 & Upregulated & P53/ERK/MAPK signalling & HBsAg, tumor size & {$[58]$} \\
\hline MEG3 & Downregulated & UHRF1/P53 & Tumor size, Edmondson grade & {$[47,59]$} \\
\hline DREH & Downregulated & $\mathrm{HBx} /$ vimentin & Tumor size, HBsAg & {$[48,49]$} \\
\hline PTENP1 & Downregulated & miR-17/miR-19b/miR-20a/AKT/PI3K signalling & Tumor size, TNM stage & {$[60]$} \\
\hline LET & Downregulated & P53/NF90/HIF- $1 \alpha$ & & {$[50]$} \\
\hline uc002mbe. 2 & Downregulated & TAS & Tumor size & {$[52]$} \\
\hline
\end{tabular}

differentiation at the tumor node metastasis stage (TNM) and cell growth processes, including cell proliferation and apoptosis, invasion, and metastasis. LncRNAs play an irreplaceable role in the progression of HCC. The key biological functions of lncRNA are related to certain signalling pathways. LncRNA expression in liver cancer tissues is associated with clinicopathological features. Deregulated lncRNAs can be a novel biomarker for diagnosing or assessing treatment efficiencies. The dysregulation of lncRNA in HCC marks a disease spectrum and is proposed to be associated with liver cancer. Many oncogenes are known as targets for liver cancer-associated lncRNAs (Table 1). LncRNA participates in HCC processes by binding to oncogenes. In addition,
lncRNA can also participate in HCC through regulatory signalling pathways, even though the underlying mechanisms are still unknown. Differential expression and potential functional roles of lncRNAs in HCC are essential.

\subsection{Upregulation of LncRNAs in HCC}

3.2.1. HOTAIR. HOTAIR, a 2158 bp lncRNA, is encoded in the HOXC locus on chromosome 12q13.1 [30]. It has been found that HOTAIR is more highly expressed in HCC tissues than in paracancerous nontumor tissues. The increased expression of HOTAIR is associated with lymph node metastasis; therefore, the HOTAIR expression level is associated 
with lymph node metastasis. Thus, the high HOTAIR levels in LT patients indicate a significantly shorter recurrencefree survival. Patients with tumors and high HOTAIR gene expression levels have a higher risk of recurrence after hepatectomy [31]. It is reported that HOTAIR promotes cell proliferation, autophagy, and invasion and reduces the response of hepatoma cells to the apoptosis stimulator TNF- $\alpha$ and the chemotherapeutic drugs cisplatin and doxorubicin [31-33]. Further studies revealed that tumorigenesis is suppressed in HCC after silencing HOTAIR, which resulted in the activation of P16 and P14 signalling via increased miR-218 expression and decreasing Bmi-1 expression, respectively. It is suggested that HOTAIR expression is associated with liver tumor differentiation, metastasis, and early recurrence [34]. These findings suggest that HOTAIR plays an important role in the development of hepatocarcinoma, so HOTAIR is the possible target for the diagnosis and treatment of liver cancer.

3.2.2. MALAT1. MALAT1 (metastasis-associated lung adenocarcinoma transcript 1 ) was initially discovered in human non-small-cell lung cancer (NSCLC). MALAT1 is the first identified metastasis-associated IncRNA, which is found upregulated in HCC cell lines and patients $[35,36]$. Functionally, MALAT1 promotes proliferation, invasion, metastasis, chemosensitivity, and autophagy in HCC cells. MALAT1 is upregulated in HCC and associated with cell proliferation and migration by regulating Bax, bcl-2, bcl-xl, caspase- 3 , and caspase-8 [36]. Moreover, MALAT1 is thought to promote proliferation during liver regeneration through the stimulation of the Wnt/catenin pathway, which is negatively regulated by p53. Silencing MALAT1 reduces cell viability, migration, and invasion and increases the sensitivity of cells to apoptotic stimuli such as cisplatin and doxorubicin, TNF-alpha, and glucose-brain toxins [37]. Thus, MALAT1 is probably involved in tumor development and could be a new biomarker for predicting tumor recurrence after LT.

3.2.3. HULC. HULC is the first identified IncRNA specifically upregulated in HCC [38]. Its coding sequence is located on chromosome 6p24.3 and is highly conserved among primates. In clinical tissues, HULC expression in HCC and hepatic colorectal metastasis samples is highly upregulated compared with that in the normal control. HULC expression is positively correlated with the Edmondson histological classification and the HBV/HBV X protein- ( $\mathrm{HBx}-)$ positive state [39-41]. Similar results were detected in plasma samples from hepatocellular carcinoma [41]. HULC promotes lipogenesis and cell proliferation, induces apoptosis and enhanced epithelial-mesenchymal transformation (EMT), and also increases the risk of liver cancer development and metastasis [40]. Previous studies illustrated that $\mathrm{HBx}-$ mediated HULC upregulation promotes the increase of mRNA and protein levels in liver cancer by downregulating the tumor suppressor gene CDKN2C (p18). CDKN2C is thought to be a tumor suppressor gene that regulates cell cycle and plays a role in signal transduction pathways, including ATM/ATR and p53 pathways. These results

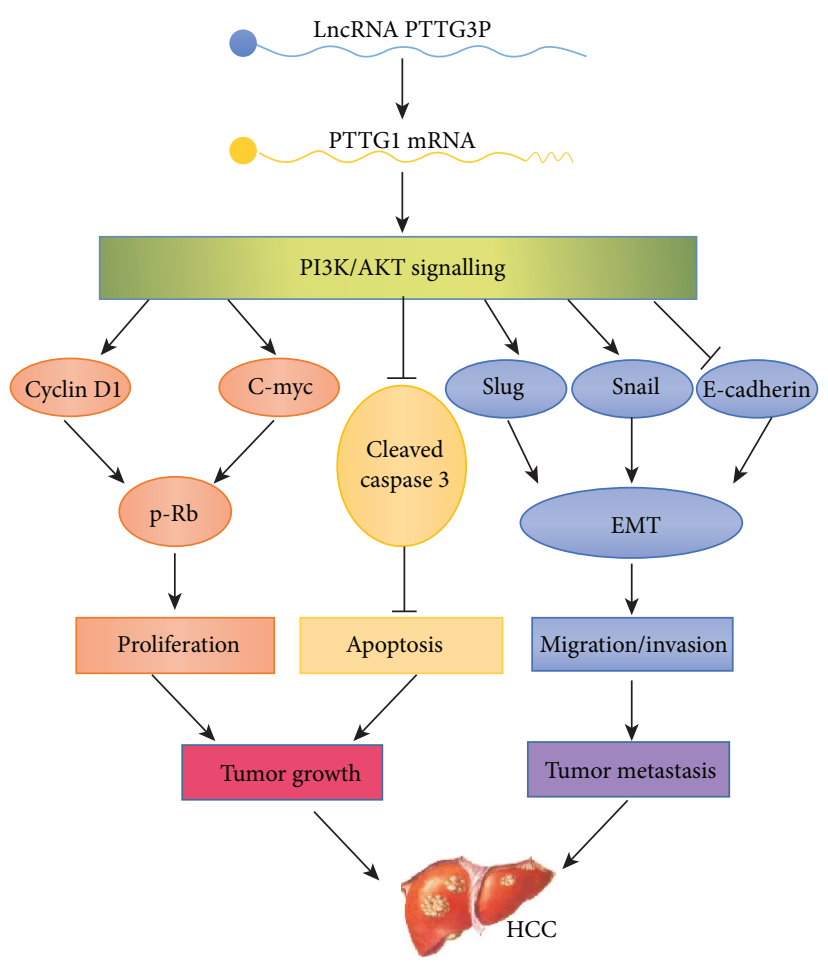

FIgURE 3: Functional diagram of lncRNA PTTG3P in HCC tumor growth and metastasis.

indicated that HULC could be used as a potential biomarker for HCC diagnosis.

3.2.4. PTTG3P. PTTG3P (pituitary tumor-transforming 3 pseudogene) is a novel lncRNA. Expression and localization of PTTG3P were analyzed using quantitative real-time polymerase chain reaction (qRTPCR) and in situ hybridization (ISH) in two patients with liver cancer [42]. It has been shown that the expression of PTTG3P in liver cancer is significantly increased. The upregulation of PTTG3P is positively correlated with a poor prognosis of liver cancer patients. PTTG3P promotes cell proliferation, inhibits apoptosis, and accelerates migration and invasion of HCC cells. Mechanically, PTTG3P is involved in tumor growth, while PTTG3P metastatic cascade promotes cells by the upregulation of PTTG1 and activation of the PI3K/AKT signalling pathway. Growth and metastasis follows, which in turn affect downstream signal transduction, through the regulation of cell cycle regulators and EMT-related factors as shown in Figure 3. Therefore, PTTG3P may be a potential target for the prevention and treatment of liver cancer [43].

3.2.5. PVT1. LncRNA PVT1 is located at 8q24.21 [44]. Studies showed that PVT1 is highly expressed in HCC tissues and associated with the number and grade of tumors. PVT1 promotes tumor growth by accelerating cell proliferation and cell cycle progression and enhancing stem cell-related properties [45]. Functionally, PVT1 can increase NOP2 levels by enhancing the stability of the NOP2 protein, and the function of PVT1 is dependent on the presence of the NOP2 protein. The hPVT1/NOP2/cell cycle gene pathway 
is involved in promoting carcinogenesis, cell proliferation, and stem cell-like properties in HCC cells [14]. Furthermore, it has been reported that PVT1 promotes proliferation, invasion, and migration of liver cancer cells by modulating the mir-150/HIG2 axis [44]. Therefore, PVT1 can be used as a diagnosis marker for HCC.

\subsection{Downregulation of LncRNAs in HCC}

3.3.1. MEG3. MEG3 is highly expressed in the human pituitary gland and is a maternal imprinting gene. The MEG3 gene is located at the imprinted DLK1-MEG3 locus on chromosome $14 q 32.3$ in humans. MEG3 expression is observed in several types of cancer [46]. MEG3 was confirmed downregulated in HCC. MEG3 regulates proliferation and apoptosis in HCC cells [47]. Mechanistically, MEG3 improves the protein stability, increases the transcriptional activity of p53 in hepatocellular carcinoma cells, and influences the expression of p53 target genes. In liver cancer tissues, MEG3 is negatively correlated with UHRF1 that plays an important role in DNA methylation by recruiting DNA methyltransferase DNMT1 during DNA replication. In the same study, UHRF1 is identified as involved in the upstream regulation of MEG3 in liver cancer by regulating DNMT1 [47]. These results indicate that MEG3 is a tumor suppressor gene and can be considered as a biomarker for liver cancer.

3.3.2. DREH. DREH is involved in HBx-mediated hepatocellular carcinoma. DREH and mouse homologous DREH were significantly downregulated in human HBV-related HCC tissues and $\mathrm{HBx}$ transgenic mice, respectively. $\mathrm{DREH}$ is a highly conserved lncRNA. The DREH reduction is significantly associated with poor survival in patients with liver cancer [48]. DREH is linked to the proliferation and metastasis of HBV-related HCC. A previous study revealed the negative correlation between Dreh expression and HBx and HBs [49]. Dreh is downregulated by HBx via the downregulation of vimentin, which results in the suppression of HCC growth and migration $[48,49]$. These results indicate that $\mathrm{DREH}$ is a tumor suppressor in the development of $\mathrm{HBx}-$ related hepatocellular carcinoma and may be a new target for the treatment of HBV-related hepatocellular carcinoma.

3.3.3. LET. "Low expression in the tumor," or LET, is present at significantly reduced levels in HCC tumor tissues and is linked to metastasis [50]. LET influences the invasiveness and metastasis of HCC cells. LET is inhibited by histone deacetylase 3 (HDAC3). LET inhibition increases the stability of nuclear factor 90 (NF90), thus promoting hypoxiainduced invasion [50]. HDAC3 induced by hypoxia inhibits lncRNA-let by reducing the lncRNA-let promoter region regulation mediated by histone acetylation. Interestingly, downregulation of lncRNA-let is a key step in stabilizing the NF90 protein, leading to hypoxic-induced infiltration of cancer cells. In addition, the relationship between hypoxia, histone acetylation disorders, low lncRNA-let expression, and metastasis has been demonstrated in clinical HCC samples. These findings demonstrated the role of lncRNA-let as a regulator of hypoxia signal transduction and provide new methods for therapeutic intervention in cancer progression.
Moreover, these findings also indicate that hypoxia can inhibit lncRNA-let expression by reducing the level of acetylation of histones $\mathrm{H} 3$ and $\mathrm{H} 4$ in its promoter region [50]. In addition, downregulation of lncRNA-let may affect the accumulation and stability of hif-1a mRNA under hypoxia.

\section{Biological Roles of LncRNAs in HCC}

4.1. Proliferation. To date, it has been proven that many lncRNAs maladjusted in hepatocellular carcinoma play a vital role in the growth of hepatocellular carcinoma in vitro or in vivo. It has been found that upregulated URHC promotes tumor and cell proliferation, which is directly related to poor prognosis in liver cancer tissues and cell lines. Further studies showed that URHC promotes cell proliferation by regulating $\mathrm{ZAK}$ protein during the activation of the ERK/MAPK signalling pathway [9]. Researchers found that H19 knockdown abolishes the tumorigenicity of HCC in vivo, and significantly, hypoxic recovery reduces growth independent of the adherent wall [51]. Moreover, the detection of the hepatoma cell line Bel7402 in vitro showed that silencing lncRNA HOTAIR could inhibit cell proliferation [31].

4.2. Apoptosis. Apoptosis is the gene-controlled autonomous and orderly death of cells. The decrease of apoptosis can promote the survival and accumulation of abnormal cells and lead to cancer development. It has been reported that lncRNA affects liver cancer by acting on apoptosis. The expression of uc $002 \mathrm{mbe} .2$ is lower in liver cancer cell tissues than in normal ones. The histone deacetylase inhibitor Trichostatin A (TSA) exerts an antitumor effect by promoting the apoptosis of liver cancer cells. Apoptosis induced by TSA is significantly inhibited by uc002mbe. 2 knockdown [52]. Thus, uc002mbe. 2 is very important in TSA-mediated hepatocyte apoptosis.

4.3. Invasion and Metastasis. The most crucial reasons for mortality and poor prognosis in patients with liver cancer are tumor metastasis and invasion, which are related to HCC both in vitro and in vivo. More and more evidences showed that lncRNA plays an important role in the invasion and metastasis of liver cancer. For example, lncRNA-related microvascular invasion in HCC (MVIH) are a class of lncRNA molecules that are highly expressed in liver cancer and are involved in angiogenesis. When the expression of these lncRNAs is high, the survival rate and prognosis are the opposite. Overexpression of MVIH in animal models promotes angiogenesis and facilitates tumor growth and metastasis. Furthermore, the expression of MVIH in liver cancer patients is significantly negatively correlated with the angiogenesis inhibitor PGK1, suggesting that MVIH promotes liver cancer metastasis by inhibiting the secretion of PGK1 [16]. In addition, recent studies indicated that lncRNA-ATB activated by TGF- $\beta$ induces EMT and cell invasion in vitro, promoting the invasion of hepatocellular carcinoma cells [53]. 


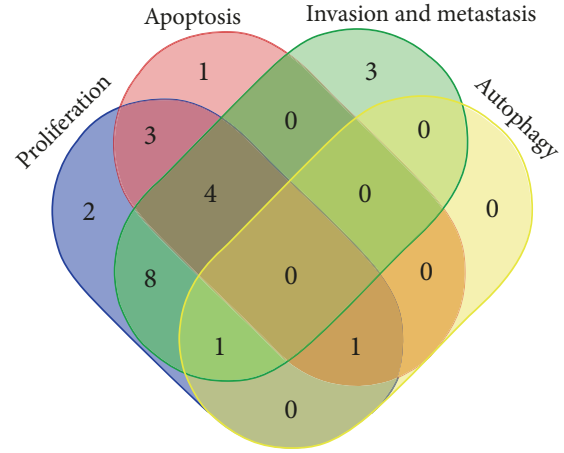

FIGURE 4: Informatics analysis of the biological functions of lncRNAs in HCC.

TABLE 2: Statistical analysis of lncRNAs and tumor biological functions.

\begin{tabular}{|c|c|c|c|}
\hline Biological functions & Number & LncRNAs & References \\
\hline \multirow{4}{*}{$\begin{array}{l}\text { Apoptosis, invasion, } \\
\text { metastasis, and proliferation }\end{array}$} & \multirow{4}{*}{4} & PTTG3P & [43] \\
\hline & & DREH & [49] \\
\hline & & ANRIL & {$[61]$} \\
\hline & & HULC & {$[53]$} \\
\hline $\begin{array}{l}\text { Apoptosis, autophagy, and } \\
\text { proliferation }\end{array}$ & 1 & PTENP1 & {$[60]$} \\
\hline $\begin{array}{l}\text { Autophagy, invasion, } \\
\text { metastasis, and proliferation }\end{array}$ & 1 & HOTAIR & {$[33]$} \\
\hline \multirow{3}{*}{ Apoptosis and proliferation } & \multirow{3}{*}{3} & uc002mbe. 2 & {$[52]$} \\
\hline & & DBH-AS1 & {$[58]$} \\
\hline & & MEG3 & [59] \\
\hline \multirow{8}{*}{$\begin{array}{l}\text { Invasion, metastasis, and } \\
\text { proliferation }\end{array}$} & \multirow{8}{*}{8} & CCAT1 & {$[62]$} \\
\hline & & HOTTIP & {$[63]$} \\
\hline & & AFAP1-AS1 & {$[64]$} \\
\hline & & UCA1 & {$[65]$} \\
\hline & & H19 & {$[55]$} \\
\hline & & XIST & [26] \\
\hline & & ZEB1-AS1 & {$[66]$} \\
\hline & & $\mathrm{HEIH}$ & {$[67]$} \\
\hline \multirow{2}{*}{ Proliferation } & \multirow{2}{*}{2} & PVT1 & {$[57]$} \\
\hline & & ROR & {$[56]$} \\
\hline Apoptosis & 1 & URHC & [9] \\
\hline \multirow{3}{*}{ Invasion and metastasis } & \multirow{3}{*}{3} & HBx-LINE1 & {$[68]$} \\
\hline & & LET & {$[50]$} \\
\hline & & $\mathrm{ATB}^{\prime}$ & [69] \\
\hline
\end{tabular}

\section{Conclusions and Future Perspectives}

In conclusion, lncRNAs play important roles in the biological processes of the occurrence, development, metastasis, and recurrence of liver cancer, which impact on the treatment and prognosis of liver cancer. Furthermore, the dysregulation of liver cancer-associated lncRNA in tumor tissues is often associated with these biological processes. LncRNA dysregulation is associated with the progression and prognosis of liver cancer. Therefore, IncRNA should a candidate biomarker for the diagnosis, prognosis, recurrence prediction, and treatment of liver cancer. The characteristics of the biological functions related to HCC IncRNA enable researchers to have a more comprehensive understanding of the occurrence of liver cancer (Figure 4, Table 2). Further research on the mechanism of lncRNA involvement in the development and progression of liver cancer is conducive to clinical diagnosis and treatment. Although a small number of lncRNAs have been studied in liver cancer, a large part of them needs to be further discovered. Therefore, further research is needed on the role and mechanism of liverspecific lncRNA in the progression of liver cancer. The knowledge of the function of lncRNA in liver cancer development is increasing, which is laying the foundation for the design of new treatment methods for liver cancer.

\section{Conflicts of Interest}

The authors declare that there is no conflict of interest regarding the publication of this paper.

\section{References}

[1] L. A. Torre, F. Bray, R. L. Siegel, J. Ferlay, J. Lortet-Tieulent, and A. Jemal, "Global cancer statistics, 2012," CA: a Cancer Journal for Clinicians, vol. 65, no. 2, pp. 87-108, 2015.

[2] H. B. El-Serag and K. L. Rudolph, "Hepatocellular carcinoma: epidemiology and molecular carcinogenesis," Gastroenterology, vol. 132, no. 7, pp. 2557-2576, 2007.

[3] R. N. Aravalli, C. J. Steer, and E. N. Cressman, "Molecular mechanisms of hepatocellular carcinoma," Hepatology, vol. 48, no. 6, pp. 2047-2063, 2008.

[4] P. Merle and C. Trepo, "Molecular mechanisms underlying hepatocellular carcinoma," Viruses, vol. 1, no. 3, pp. 852872, 2009.

[5] F. J. Yu, J. J. Zheng, P. H. Dong, and X. M. Fan, "Long non-coding RNAs and hepatocellular carcinoma (Review)," Molecular and Clinical Oncology, vol. 3, no. 1, pp. 13-17, 2015.

[6] C. P. Ponting, P. L. Oliver, and W. Reik, "Evolution and functions of long noncoding RNAs," Cell, vol. 136, no. 4, pp. 629-641, 2009.

[7] P. G. Mansourian, M. Yoneda, M. Krishna Rao, F. J. Martinez, E. Thomas, and E. R. Schiff, "Effects of statins on the risk of hepatocellular carcinoma," Gastroenterology \& Hepatology, vol. 10, no. 7, pp. 417-426, 2014.

[8] M. D. Huang, W. M. Chen, F. Z. Qi et al., "Long non-coding RNA ANRIL is upregulated in hepatocellular carcinoma and regulates cell proliferation by epigenetic silencing of KLF2," Journal of Hematology \& Oncology, vol. 8, no. 1, p. 57, 2015.

[9] W. H. Xu, J. B. Zhang, Z. Dang et al., "Long non-coding RNA URHC regulates cell proliferation and apoptosis via ZAK through the ERK/MAPK signaling pathway in hepatocellular carcinoma," International Journal of Biological Sciences, vol. 10, no. 7, pp. 664-676, 2014.

[10] M. C. Tsai, O. Manor, Y. Wan et al., "Long noncoding RNA as modular scaffold of histone modification complexes," Science, vol. 329, no. 5992, pp. 689-693, 2010. 
[11] J. L. Rinn and H. Y. Chang, "Genome regulation by long noncoding RNAs," Annual Review of Biochemistry, vol. 81, no. 1, pp. 145-166, 2012.

[12] K. C. Wang and H. Y. Chang, "Molecular mechanisms of long noncoding RNAs," Molecular Cell, vol. 43, no. 6, pp. 904-914, 2011.

[13] Y. Li, S. D. Egranov, L. Yang, and C. Lin, "Molecular mechanisms of long noncoding RNAs-mediated cancer metastasis," Genes, Chromosomes \& Cancer, vol. 58, no. 4, pp. 200-207, 2018.

[14] F. Wang, J. H. Yuan, S. B. Wang et al., "Oncofetal long noncoding RNA PVT1 promotes proliferation and stem cell-like property of hepatocellular carcinoma cells by stabilizing NOP2," Hepatology, vol. 60, no. 4, pp. 1278-1290, 2014.

[15] F. Yang, L. Zhang, X. S. Huo et al., "Long noncoding RNA high expression in hepatocellular carcinoma facilitates tumor growth through enhancer of zeste homolog 2 in humans," Hepatology, vol. 54, no. 5, pp. 1679-1689, 2011.

[16] S. X. Yuan, F. Yang, Y. Yang et al., "Long noncoding RNA associated with microvascular invasion in hepatocellular carcinoma promotes angiogenesis and serves as a predictor for hepatocellular carcinoma patients' poor recurrence-free survival after hepatectomy," Hepatology, vol. 56, no. 6, pp. 2231-2241, 2012.

[17] A. Sahu, U. Singhal, and A. M. Chinnaiyan, "Long noncoding RNAs in cancer: from function to translation," Trends in Cancer, vol. 1, no. 2, pp. 93-109, 2015.

[18] K. Arab, Y. J. Park, A. M. Lindroth et al., "Long noncoding RNA TARID directs demethylation and activation of the tumor suppressor TCF21 via GADD45A," Molecular Cell, vol. 55, no. 4, pp. 604-614, 2014.

[19] R. Taby and J. P. Issa, "Cancer epigenetics," CA: a Cancer Journal for Clinicians, vol. 60, no. 6, pp. 376-392, 2010.

[20] Y. Wang, L. He, Y. Du et al., "The long noncoding RNA IncTCF7 promotes self-renewal of human liver cancer stem cells through activation of Wnt signaling," Cell Stem Cell, vol. 16, no. 4, pp. 413-425, 2015.

[21] M. Nakayama and M. Oshima, "Mutant p53 in colon cancer," Journal of Molecular Cell Biology, vol. 11, no. 4, pp. 267-276, 2018.

[22] A. Vazquez, E. E. Bond, A. J. Levine, and G. L. Bond, "The genetics of the p53 pathway, apoptosis and cancer therapy," Nature Reviews Drug Discovery, vol. 7, no. 12, pp. 979-987, 2008.

[23] N. Dimitrova, J. R. Zamudio, R. M. Jong et al., "LincRNA-p21 activates p21 in cis to promote polycomb target gene expression and to enforce the G1/S checkpoint," Molecular Cell, vol. 54, no. 5, pp. 777-790, 2014.

[24] M. V. Iorio and C. M. Croce, "MicroRNA dysregulation in cancer: diagnostics, monitoring and therapeutics. A comprehensive review," EMBO Molecular Medicine, vol. 9, no. 6, p. 852, 2017.

[25] L. Salmena, L. Poliseno, Y. Tay, L. Kats, and P. P. Pandolfi, "A ceRNA hypothesis: the Rosetta Stone of a hidden RNA language?," Cell, vol. 146, no. 3, pp. 353-358, 2011.

[26] Y. Mo, Y. Lu, P. Wang et al., "Long non-coding RNA XIST promotes cell growth by regulating miR-139-5p/PDK1/AKT axis in hepatocellular carcinoma," Tumour biology : the journal of the International Society for Oncodevelopmental Biology and Medicine, vol. 39, no. 2, article 1010428317690999, 2017.
[27] S. Colak and P. Ten Dijke, "Targeting TGF- $\beta$ signaling in cancer," Trends in Cancer, vol. 3, no. 1, pp. 56-71, 2017.

[28] J. Taipale and P. A. Beachy, "The Hedgehog and Wnt signalling pathways in cancer," Nature, vol. 411, no. 6835, pp. 349-354, 2001.

[29] F. Liu and S. E. Millar, "Wnt/beta-catenin signaling in oral tissue development and disease," Journal of Dental Research, vol. 89, no. 4, pp. 318-330, 2010.

[30] J. Zhang, P. Zhang, L. Wang, H. L. Piao, and L. Ma, "Long noncoding RNA HOTAIR in carcinogenesis and metastasis," Acta Biochimica et Biophysica Sinica, vol. 46, no. 1, pp. 1-5, 2014.

[31] Y. J. Geng, S. L. Xie, Q. Li, J. Ma, and G. Y. Wang, "Large intervening non-coding RNA HOTAIR is associated with hepatocellular carcinoma progression," The Journal of International Medical Research, vol. 39, no. 6, pp. 2119-2128, 2011.

[32] W. M. Fu, X. Zhu, W. M. Wang et al., "Hotair mediates hepatocarcinogenesis through suppressing miRNA-218 expression and activating P14 and P16 signaling," Journal of Hepatology, vol. 63, no. 4, pp. 886-895, 2015.

[33] E. Li, Z. Zhao, B. Ma, and J. Zhang, "Long noncoding RNA HOTAIR promotes the proliferation and metastasis of osteosarcoma cells through the AKT/mTOR signaling pathway," Experimental and Therapeutic Medicine, vol. 14, no. 6, pp. 5321-5328, 2017.

[34] Z. Yang, L. Zhou, L. M. Wu et al., "Overexpression of long non-coding RNA HOTAIR predicts tumor recurrence in hepatocellular carcinoma patients following liver transplantation," Annals of Surgical Oncology, vol. 18, no. 5, pp. 12431250, 2011.

[35] R. Lin, S. Maeda, C. Liu, M. Karin, and T. S. Edgington, “A large noncoding RNA is a marker for murine hepatocellular carcinomas and a spectrum of human carcinomas," Oncogene, vol. 26, no. 6, pp. 851-858, 2007.

[36] M. C. Lai, Z. Yang, L. Zhou et al., "Long non-coding RNA MALAT-1 overexpression predicts tumor recurrence of hepatocellular carcinoma after liver transplantation," Medical Oncology, vol. 29, no. 3, pp. 1810-1816, 2012.

[37] C. Li, L. Chang, Z. Chen, Z. Liu, Y. Wang, and Q. Ye, “The role of lncRNA MALAT1 in the regulation of hepatocyte proliferation during liver regeneration," International Journal of Molecular Medicine, vol. 39, no. 2, pp. 347-356, 2017.

[38] K. Panzitt, M. M. Tschernatsch, C. Guelly et al., "Characterization of HULC, a novel gene with striking up-regulation in hepatocellular carcinoma, as noncoding RNA," Gastroenterology, vol. 132, no. 1, pp. 330-342, 2007.

[39] H. Xie, H. Ma, and D. Zhou, "Plasma HULC as a promising novel biomarker for the detection of hepatocellular carcinoma," BioMed Research International, vol. 2013, Article ID 136106, 5 pages, 2013.

[40] Y. Du, G. Kong, X. You et al., "Elevation of highly up-regulated in liver cancer (HULC) by hepatitis B virus X protein promotes hepatoma cell proliferation via down-regulating p18," The Journal of Biological Chemistry, vol. 287, no. 31, pp. 2630226311, 2012.

[41] I. J. Matouk, I. Abbasi, A. Hochberg, E. Galun, H. Dweik, and M. Akkawi, "Highly upregulated in liver cancer noncoding RNA is overexpressed in hepatic colorectal metastasis," European Journal of Gastroenterology \& Hepatology, vol. 21, no. 6, pp. 688-692, 2009.

[42] W. Weng, S. Ni, Y. Wang et al., "PTTG3P promotes gastric tumour cell proliferation and invasion and is an indicator of 
poor prognosis," Journal of Cellular and Molecular Medicine, vol. 21, no. 12, pp. 3360-3371, 2017.

[43] J. L. Huang, S. W. Cao, Q. S. Ou et al., "The long non-coding RNA PTTG3P promotes cell growth and metastasis via upregulating PTTG1 and activating PI3K/AKT signaling in hepatocellular carcinoma," Molecular Cancer, vol. 17, no. 1, p. 93, 2018.

[44] Y. Xu, X. Luo, W. He et al., "Long non-coding RNA PVT1/miR-150/HIG2 axis regulates the proliferation, invasion and the balance of iron metabolism of hepatocellular carcinoma," Cellular Physiology and Biochemistry : International Journal of Experimental Cellular Physiology, Biochemistry, and Pharmacology, vol. 49, no. 4, pp. 1403-1419, 2018.

[45] Y. Zhang, W. J. Mo, X. Wang et al., "Microarray-based bioinformatics analysis of the prospective target gene network of key miRNAs influenced by long non-coding RNA PVT1 in HCC," Oncology Reports, vol. 40, no. 1, pp. 226240, 2018.

[46] L. Benetatos, G. Vartholomatos, and E. Hatzimichael, "MEG3 imprinted gene contribution in tumorigenesis," International Journal of Cancer, vol. 129, no. 4, pp. 773-779, 2011.

[47] J. Zhu, S. Liu, F. Ye et al., "Long noncoding RNA MEG3 interacts with p53 protein and regulates partial p53 target genes in hepatoma cells," PLoS One, vol. 10, no. 10, article e0139790, 2015.

[48] J. F. Huang, Y. J. Guo, C. X. Zhao et al., "Hepatitis B virus X protein $(\mathrm{HBx})$-related long noncoding RNA (lncRNA) down-regulated expression by $\mathrm{HBx}$ (Dreh) inhibits hepatocellular carcinoma metastasis by targeting the intermediate filament protein vimentin," Hepatology, vol. 57 , no. 5, pp. $1882-$ 1892, 2013.

[49] D. Lv, Y. Wang, Y. Zhang, P. Cui, and Y. Xu, "Downregulated long non-coding RNA DREH promotes cell proliferation in hepatitis B virus-associated hepatocellular carcinoma," Oncology Letters, vol. 14, no. 2, pp. 2025-2032, 2017.

[50] F. Yang, X. S. Huo, S. X. Yuan et al., "Repression of the long noncoding RNA-LET by histone deacetylase 3 contributes to hypoxia-mediated metastasis," Molecular Cell, vol. 49, no. 6, pp. 1083-1096, 2013.

[51] I. J. Matouk, N. DeGroot, S. Mezan et al., "The H19 noncoding RNA is essential for human tumor growth," PLoS One, vol. 2, no. 9, article e845, 2007.

[52] H. Yang, Y. Zhong, H. Xie et al., "Induction of the liver cancerdown-regulated long noncoding RNA uc002mbe. 2 mediates trichostatin-induced apoptosis of liver cancer cells," Biochemical Pharmacology, vol. 85, no. 12, pp. 1761-1769, 2013.

[53] H. Xiong, B. Li, J. He, Y. Zeng, Y. Zhang, and F. He, "IncRNA HULC promotes the growth of hepatocellular carcinoma cells via stabilizing COX-2 protein," Biochemical and Biophysical Research Communications, vol. 490, no. 3, pp. 693-699, 2017.

[54] X. Xin, M. Wu, Q. Meng et al., "Long noncoding RNA HULC accelerates liver cancer by inhibiting PTEN via autophagy cooperation to miR15a," Molecular Cancer, vol. 17, no. 1, p. 94, 2018.

[55] M. L. Yang, Z. Huang, Q. Wang et al., "The association of polymorphisms in lncRNA-H19 with hepatocellular cancer risk and prognosis," Bioscience Reports, vol. 38, no. 5, article BSR20171652, 2018.

[56] A. Zhang, N. Zhou, J. Huang et al., "The human long noncoding RNA-RoR is a p53 repressor in response to DNA damage," Cell Research, vol. 23, no. 3, pp. 340-350, 2013.
[57] J. Guo, C. Hao, C. Wang, and L. Li, "Long noncoding RNA PVT1 modulates hepatocellular carcinoma cell proliferation and apoptosis by recruiting EZH2," Cancer Cell International, vol. 18, no. 1, p. 98, 2018.

[58] J. Bao, X. Chen, Y. Hou, G. Kang, Q. Li, and Y. Xu, "LncRNA DBH-AS1 facilitates the tumorigenesis of hepatocellular carcinoma by targeting miR-138 via FAK/Src/ERK pathway," Biomedicine \& Pharmacotherapy, vol. 107, pp. 824-833, 2018.

[59] H. Zhuo, J. Tang, Z. Lin et al., "The aberrant expression of MEG3 regulated by UHRF1 predicts the prognosis of hepatocellular carcinoma," Molecular Carcinogenesis, vol. 55, no. 2, pp. 209-219, 2016.

[60] C. L. Chen, Y. W. Tseng, J. C. Wu et al., "Suppression of hepatocellular carcinoma by baculovirus-mediated expression of long non-coding RNA PTENP1 and microRNA regulation," Biomaterials, vol. 44, pp. 71-81, 2015.

[61] J. Ma, T. Li, X. Han, and H. Yuan, "Knockdown of lncRNA ANRIL suppresses cell proliferation, metastasis, and invasion via regulating miR-122-5p expression in hepatocellular carcinoma," Journal of Cancer Research and Clinical Oncology, vol. 144, no. 2, pp. 205-214, 2018.

[62] H. Zhu, X. Zhou, H. Chang et al., "CCAT1 promotes hepatocellular carcinoma cell proliferation and invasion," International Journal of Clinical \& Experimental Pathology, vol. 8, no. 5, pp. 5427-5434, 2015.

[63] F. H. Tsang, S. L. Au, L. Wei et al., "Long non-coding RNA HOTTIP is frequently up-regulated in hepatocellular carcinoma and is targeted by tumour suppressive miR-125b," Liver International, vol. 35, no. 5, pp. 1597-1606, 2015.

[64] J. Y. Zhang, M. Z. Weng, F. B. Song et al., "Long noncoding RNA AFAP1-AS1 indicates a poor prognosis of hepatocellular carcinoma and promotes cell proliferation and invasion via upregulation of the RhoA/Rac2 signaling," International Journal of Oncology, vol. 48, no. 4, pp. 1590-1598, 2016.

[65] J. J. Hu, W. Song, S. D. Zhang et al., "HBx-upregulated lncRNA UCA1 promotes cell growth and tumorigenesis by recruiting EZH2 and repressing p27Kip1/CDK2 signaling," Scientific Reports, vol. 6, no. 1, article 23521, 2016.

[66] T. Li, J. Xie, C. Shen et al., "Upregulation of long noncoding RNA ZEB1-AS1 promotes tumor metastasis and predicts poor prognosis in hepatocellular carcinoma," Oncogene, vol. 35, no. 12, pp. 1575-1584, 2016.

[67] Y. Zhang, Z. Li, Y. Zhang, Q. Zhong, Q. Chen, and L. Zhang, "Molecular mechanism of HEIH and HULC in the proliferation and invasion of hepatoma cells," International Journal of Clinical and Experimental Medicine, vol. 8, no. 8, pp. 1295612962, 2015.

[68] H. W. Liang, N. Wang, Y. Wang et al., "Hepatitis B virushuman chimeric transcript HBx-LINE1 promotes hepatic injury via sequestering cellular microRNA-122," Journal of Hepatology, vol. 64, no. 2, pp. 278-291, 2016.

[69] Y. Li, Y. Ye, and H. Chen, "Astragaloside IV inhibits cell migration and viability of hepatocellular carcinoma cells via suppressing long noncoding RNA ATB," Biomedicine \& Pharmacotherapy, vol. 99, pp. 134-141, 2018. 


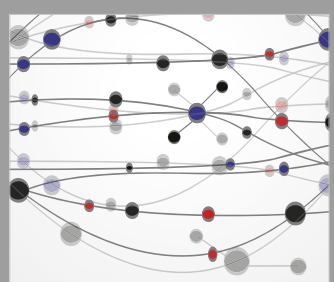

The Scientific World Journal
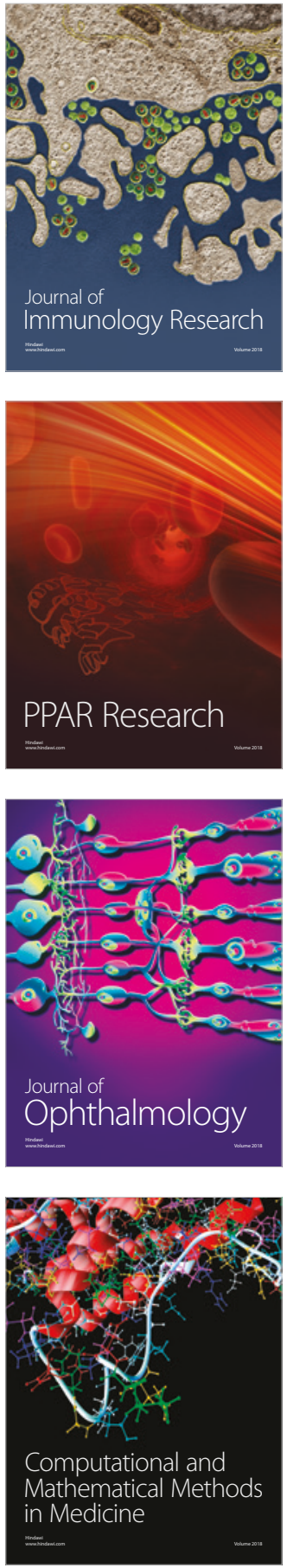

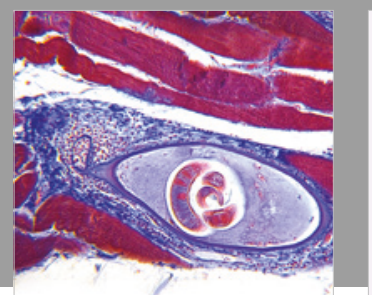

Gastroenterology Research and Practice

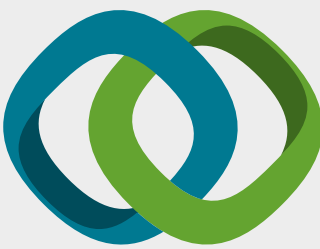

\section{Hindawi}

Submit your manuscripts at

www.hindawi.com
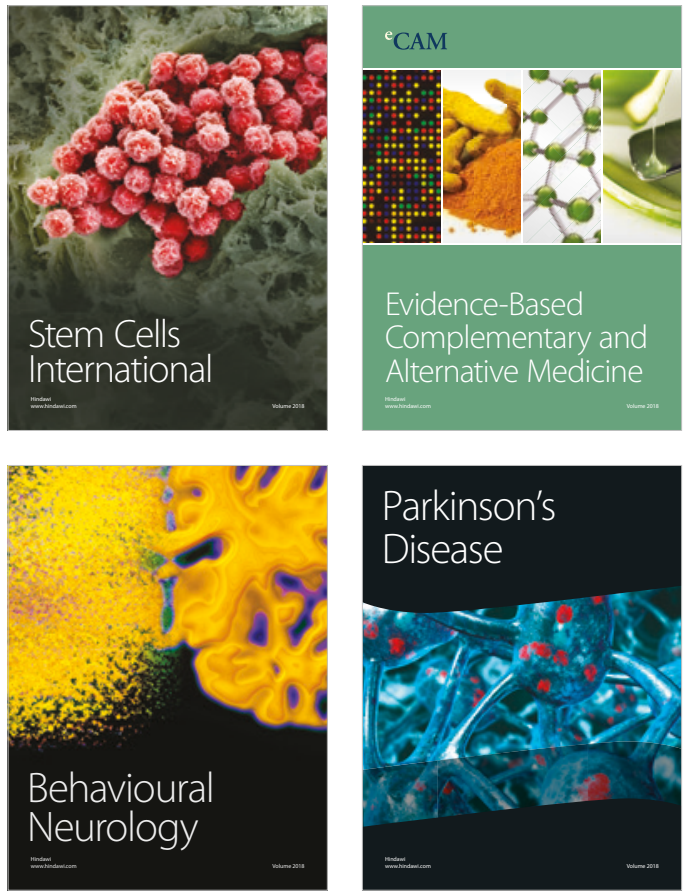

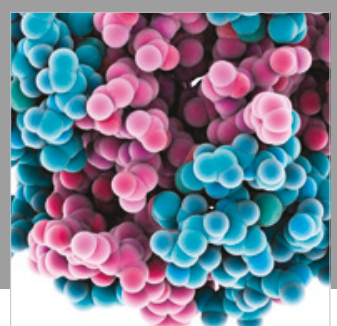

ournal of

Diabetes Research

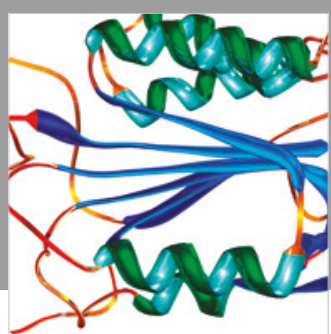

Disease Markers
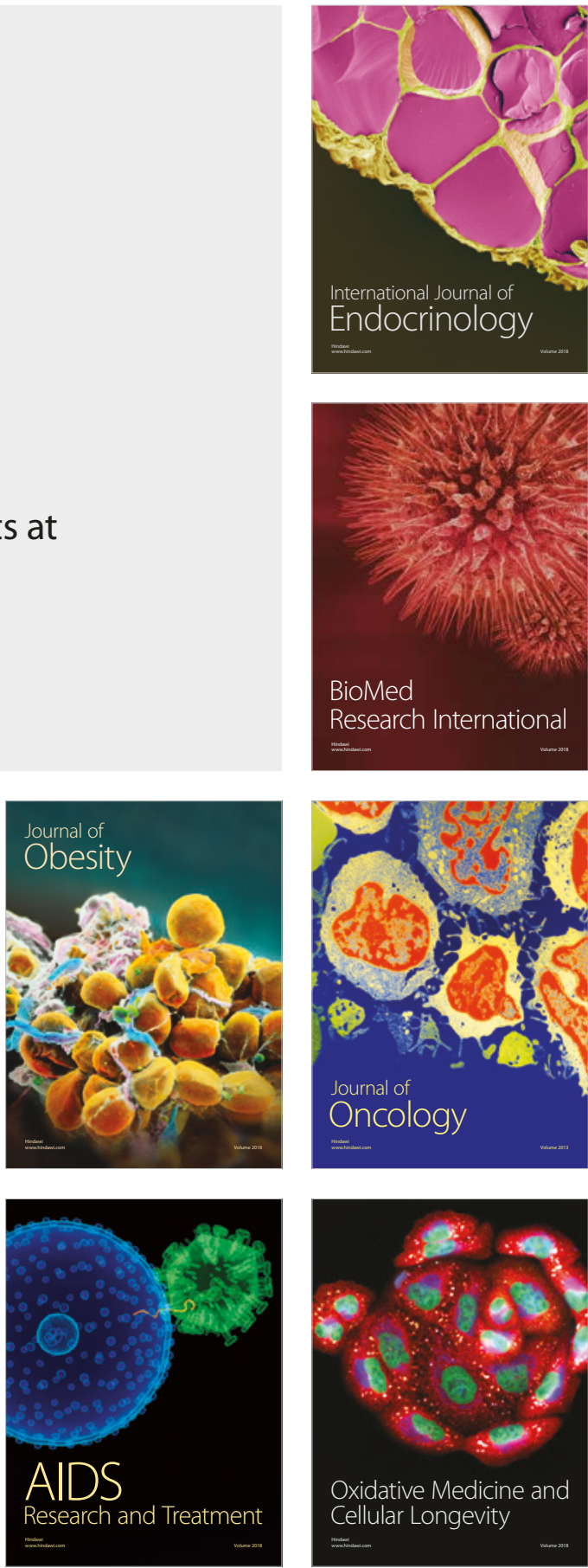